Published in final edited form as:

J Med Chem. 2007 November 1; 50(22): 5324-5329. doi:10.1021/jm070683u.

\title{
From Docking False-Positive to Active Anti-HIV Agent
}

\author{
Gabriela Barreiro ${ }^{\dagger}$, Joseph T. $\operatorname{Kim}^{\dagger}$, Cristiano R. W. Guimarães ${ }^{\dagger}$, Christopher M. Bailey $\ddagger$, \\ Robert A. Domaoal $\ddagger$, Ligong Wang $\mp$, Karen S. Anderson $\ddagger$, and William L. Jorgensen $\dagger$ \\ $\dagger$ Contribution from the Department of Chemistry, Yale University, New Haven, Connecticut 06520-8107 \\ $\$$ The Department of Pharmacology, Yale University School of Medicine, New Haven, CT 06520-8066
}

\section{Abstract}

\begin{abstract}
Virtual screening of the Maybridge library of ca. 70,000 compounds was performed using a similarity filter, docking, and MM-GB/SA post-processing to seek potential non-nucleoside inhibitors of HIV-1 reverse transcriptase (NNRTIs). Though known NNRTIs were retrieved well, purchase and assaying of representative, top-scoring compounds from the library failed to yield any active anti-HIV agents. However, the highest-ranked library compound, oxadiazole 1, was pursued as a potential "near-miss" with the $B O M B$ program to seek constructive modifications. Subsequent synthesis and assaying of several polychloro-analogs did yield anti-HIV agents with $\mathrm{EC}_{50}$ values as low as $310 \mathrm{nM}$. The study demonstrates that it is possible to learn from a formally unsuccessful virtual-screening exercise and, with the aid of computational analyses, to evolve efficiently a false positive into a true active. In addition, the need for adequate structure validation was confirmed by the apparent misrepresentation of a purchased compound elsewhere as the present oxadiazole core compound, $\mathbf{1 6 .}$
\end{abstract}

\section{Introduction}

The AIDS crisis continues with ca. 40 million people infected by HIV and 2.9 million HIVrelated deaths in $2006 .{ }^{1}$ The virally encoded proteins of HIV provide chemotherapeutic targets for the treatment of infection by the virus. A principal point of attack has been HIV reverse transcriptase (RT), which is required for the conversion of the viral genomic RNA to DNA and for successful infection of host cells. This has led to the development and FDA approval of two important classes of anti-HIV drugs: (i) nucleoside and nucleotide RT inhibitors (NRTIs and NtRTIs), e.g., AZT, ddI, ddC, d4T, FTC, TDF, and 3TC, and (ii) non-nucleoside RT inhibitors (NNRTIs), specifically, nevirapine, delavirdine, and efavirenz. The NRTIs and NtRTIs are actually faulty substrates that cause premature termination of the growing DNA transcript, while NNRTIs are true inhibitors which bind to an allosteric pocket in the vicinity of the polymerase active site. ${ }^{2}$ The therapeutic situation is challenged by rapid mutation of the virus to yield resistant strains. This leads to need for new drugs with activity against at least parts of the spectrum of variants, which are now clinically observed.

Our own efforts have been directed at the development of NNRTIs with enhanced therapeutic spectra and auspicious pharmacological properties. The approach to-date has featured focused synthetic organic chemistry and anti-HIV assaying driven by automated procedures for creation and evaluation of virtual libraries, estimation of pharmacological properties, and lead

Correspondence to: William L. Jorgensen, william. jorgensen@yale . edu.

aAbbreviations: HIV, human immunodeficiency virus; AIDS, acquired immunodeficiency syndrome; HIV-RT, HIV reverse transcriptase; NRTI, nucleoside inhibitor of HIV-RT; NNRTI, non-nucleoside inhibitor of HIV-RT; MM-GB/SA, molecular mechanics - generalized Born/surface area; OPLS, optimized potentials for liquid simulations; OPLS-AA, OPLS all-atom; CM1 A, charge model $1 \mathrm{~A} ; \mathrm{QP} \log \mathrm{P}_{\mathrm{o} / \mathrm{w}}, \log$ of the octanol/water partition coefficient predicted by QikProp; THF, tetrahydrofuran; CDI, 1,1'carbonyldiimidazole 
optimization featuring free-energy perturbation calculations to assess relative protein-ligand binding affinities. ${ }^{3}$ Highly potent and structurally diverse anti-HIV agents have been discovered, ${ }^{3}$ however, we continue to seek activity against an ever-wider range of viral mutants and exploration of alternative structural classes for NNRTIs. To this end, reported successes for lead generation by molecular docking have been intriguing, ${ }^{4}$ and it was decided to try this approach to seek novel NNRTIs. The following report provides a case study on a common dilemma in a virtual or high-throughput screening exercise. It is demonstrated that with confidence in computed structures and estimated activities, it is possible to convert a false positive into an active agent.

\section{Virtual Screening}

A summary of the virtual screening effort is presented here with full details provided elsewhere.

5 Virtual screening was performed on roughly 70,000 compounds from the Maybridge collection, which was supplemented with 26 known NNRTIs. The screening protocol began with a similarity filter that retrieves $60 \%$ of the known actives in the top $5 \%$ of the screened library. The ca. 2000 library compounds that were most similar to the known actives were then docked into the structure of wild-type HIV-1 RT, taken from its $1 \mathrm{rt} 4$ crystal structure ${ }^{6}$ in complex with a carboxanilide NNRTI, using Glide 3.5 with standard precision. ${ }^{7}$ The top 500 compounds were then re-docked and scored in Glide extra-precision (XP) mode. The top 100 of these were post-scored with an MM-GB/SA method that was shown to provide high correlation between predicted and observed activities for known NNRTIs. 5 The MM-GB/SA procedure included a conformational search for the ligand and optimization of the complex with the OPLS-AA force field 8 as well as evaluation of the change in free energy of hydration using the Generalized Born/Surface Area approach, ${ }^{9}$ as implemented in MacroModel. ${ }^{10}$

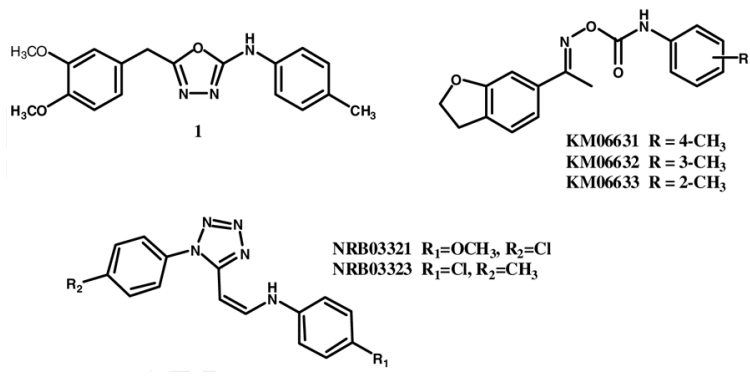

The final top-20 ranked compounds consisted of 10 library compounds and 10 known NNRTIs. The best ranked library compound, 1 (S10087), was third overall and the other top-20 library compounds were in two series containing a dihydrobenzofuran or 1-phenyltetrazole. The known NNRTIs are 2 (SDABO-3w), 3 (UC781), 4 (TMC125), 5 (efavirenz) and three analogs, and three pyrimidine derivatives from our group. ${ }^{3}$ Six of the top library compounds were purchased from Maybridge, 1, and the KM and NRB compounds shown above. The two NRB compounds were found to be impure and gave mass spectra that are inconsistent with the illustrated structures. The other four library compounds gave correct NMR and mass spectra, were purified by HPLC, recrystallized, and submitted for assay. Activities against the IIIB strain of HIV-1 were determined using MT-2 human T-cells; EC $_{50}$ values are obtained as the dose required to achieve $50 \%$ protection of the infected cells using the MTT colorimetric method. The $\mathrm{CC}_{50}$ for inhibition of MT-2 cell growth by $50 \%$ is obtained simultaneously. 11 The four compounds showed no anti-HIV activity up to their $\mathrm{CC}_{50}$ concentrations of 61,41 , 75 , and $3 \mu \mathrm{M}$, respectively. 

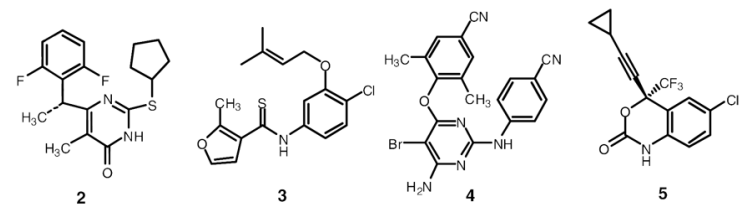

In essence, our best effort was made to screen computationally the Maybridge collection in search of NNRTI leads. The screening protocol seemed well-founded in view of (a) the testing of the MM-GB/SA procedure, (b) Glide-docked structures ("poses") for nine known NNRTIs agreed well with crystallographic results, and (c) the NRB compounds are close in structure to recently reported NNRTI HTS-hits that feature $\mathrm{N}$-phenyl triazoles and tetrazoles (Scheme 1). ${ }^{12}$

In fact, 16 compounds had been purchased from Maybridge and assayed from a preliminary version of the same docking exercise that did not include the MM-GB/SA post-scoring. These compounds were also all inactive in the MT-2 assay, and structure visualization indicated that there were generosities in the evaluation of conformational energetics by Glide including some twisted groups such as amides that should be more-or-less planar. This led to exploration of the post-scoring and to report of an analysis of the current problems for rank-ordering of compounds by docking owing to the uncertainties in evaluating conformational energetics and "conformer focusing", basically the need to evaluate the overall change in conformational energetics for the ligand and protein between the unbound and bound states. ${ }^{13}$ In the end, the structures from the docking plus MM-energy minimization appear reasonable, as illustrated in Figure 1 for $\mathbf{1}$, and the penalties for achieving the docked conformations were not high.

\section{Post Mortem}

Several reasons can be suggested for the lack of success with finding active compounds in the Maybridge library: (a) remaining inaccuracies in the scoring including the "conformer focusing" problem and lack of explicit representation of the solvent, (b) the size of the screened library - only 70,000 compounds, (c) needing to dig deeper into the deck (purchase more compounds), and (d) the sensitivity of activity to structure. Concerning (d), as demonstrated in our own NNRTI series, addition of a chloro and a methoxy group takes 6 from an $\mathrm{EC}_{50}$ of $30 \mu \mathrm{M}$ to $10 \mathrm{nM}(\mathbf{8}),{ }^{3}$ while addition of another small group in the wrong place can be very detrimental $(\mathbf{9}$ or $\mathbf{1 0})$
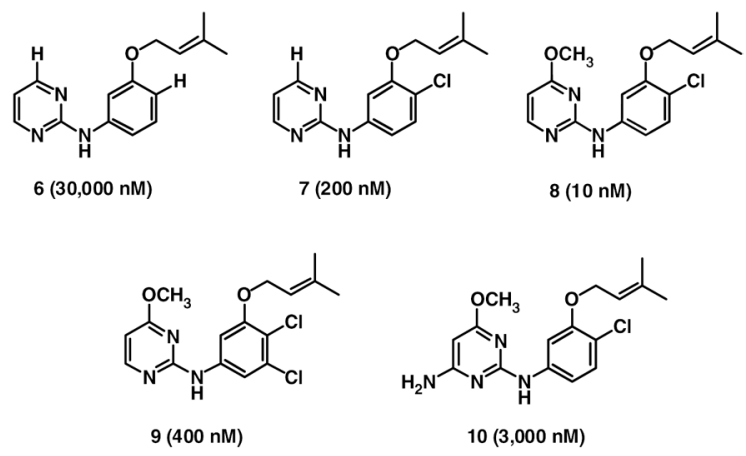

Thus, it is expected that there can be "near-misses" from virtual or experimental screening in which the activity window for an assay is missed owing to a seemingly minor structural omission or addition. Even given a viable core, the chance of an active substituent pattern for a target being included in a molecular library is extremely low. ${ }^{14}$ Of course, the nature of the assay can also be an issue. It is possible that some inactive compounds in a cell-based assay 
might be active in an in vitro assay for enzyme inhibition. However, in the present case, good correlations have been observed consistently for NNRTIs in assays for inhibition of reverse transcription and in cell-based anti-HIV analyses. ${ }^{15}$ Furthermore, the value of a lead that was not active in the cell-based assay would be comparatively low.

\section{From False-Positive to Lead}

The possibility that the virtual screening had provided a near-miss was pursued to ascertain if a false-positive could be evolved into a lead and to determine how close the miss was in structure to a true active. The oxadiazole motif was chosen since 1 ranked so highly in the virtual screening. The predicted structure of the complex (Figure 1) seemed reasonable and reminiscent of the structures of complexes of HIV-RT with analogs of 4. ${ }^{16,17}$ Three analogs of 1 differing in the substitution of the anilinyl ring were also in the top-50 ranked compounds: 3-fluoro (S10076), 3-chloro, 4-methyl (S10085), and 2,4-dichloro (S10089). All four compounds share the 3,4-dimethoxybenzyl group. Suspicion centered on this subunit and on the oxadiazole ring itself. $\mathbf{1}$ and $\mathbf{4}$ can be viewed as members of a general structural class, UHet-NH-PhX, ${ }^{3 \mathrm{a}}$ where $\mathrm{U}$ is an unsaturated group such as a substituted benzyl, anilinyl, or phenoxy group, Het is a heterocycle, and PhX is a substituted phenyl group. ${ }^{18}$ NNRTIs have been reported by Janssen and co-workers where Het is a pyrimidine, triazine, or pyrazinone. 19,20 However, there have not been reports where $\mathrm{U}$ is a 3,4-disubstituted benzyl group or Het is a 5-membered-ring heterocyle. Finally, the para-methyl substituent in $\mathbf{1}$ was not viewed as optimal; chloro and cyano are common in the Janssen NNRTIs, and in our series 4-chloro and cyano are preferred over methyl by a factor of ca. 15 in $\mathrm{EC}_{50}$ values. ${ }^{3}$ Thus, surgery was indicated for both phenyl rings of $\mathbf{1}$, though it was possible that no oxadiazole derivative would be viable.

\section{BOMB Analyses}

At this point a full substituent scan was indicated for replacement of each phenyl hydrogen in the 2-anilinyl-5-benzyloxadiazole core. This is carried out with the BOMB program, which creates analogs by adding substituents to a core that has been placed in a binding site. ${ }^{3 \mathrm{a}} \mathrm{A}$ thorough conformational search is performed for each analog and the position, orientation and dihedral angles for the analog are optimized using the OPLS-AA force field for the protein and OPLS/CM1A for the analog. ${ }^{8}$ The protein is rigid except for optimization of the terminal dihedral angles for side chains with hydrogen-bonding groups, e.g., the $\mathrm{OH}$ of tyrosine and carboxylate group of aspartate. The resultant conformer for each analog with the lowest energy is then evaluated with a docking-like scoring function. The current scoring function has been trained to reproduce experimental activity data for 339 complexes of HIV-RT, COX-2, FK506 binding protein (FKBP), and p38 kinase (Figure 2). The scoring function only contains five descriptors that were obtained by linear regression: the octanol/water partition coefficient for the analog as computed by QikProp $\left(\mathrm{QP} \log \mathrm{P}_{\mathrm{o} / \mathrm{w}}\right),{ }^{21}$ the amount of hydrophobic surface area for the protein that is buried upon complex formation $\left(\triangle \mathrm{FOSA}_{\mathrm{P}}\right)$, the number of potential hydrogen-bond donating hydrogens in the analog $\left(\mathrm{HBDN}_{\mathrm{L}}\right)$, the number of non-conjugated amides in the analog, and the number of "bad" protein-analog contacts in the complex (NBAD). The latter represent structural mismatches between two atoms within $4 \AA$, typically between a potential hydrogen-bonding oxygen or nitrogen and a saturated carbon atom or between a potential hydrogen-bond accepting $\mathrm{O}$ or $\mathrm{N}$ and another such atom or an aryl carbon atom.

Interestingly, (a) the most significant descriptor is $\mathrm{QP} \log \mathrm{P}_{\mathrm{o} / \mathrm{w}}$, which alone yields a fit with an $r^{2}$ of 0.47 , and (b) inclusion of energetic results from full conjugate-gradient optimizations of the complexes created by $B O M B$ was found to have little impact on the accuracy of the scoring. Though the $B O M B$ scoring is still under development, the current version provides a useful evaluation of potential activity (Figure 2). 
A standard protocol for a substituent scan with $B O M B$ is to replace each hydrogen of a core with 10 small groups that have been selected for difference in size, electronic character, and hydrogen-bonding patterns: $\mathrm{Cl}, \mathrm{CH}_{3}, \mathrm{NH}_{2}, \mathrm{OH}, \mathrm{CH}_{2} \mathrm{NH}_{2}, \mathrm{CH}_{2} \mathrm{OH}, \mathrm{CHO}, \mathrm{CN}, \mathrm{NHCH}_{3}$, and $\mathrm{OCH}_{3}$. To begin, the structure of $\mathbf{1}$ bound to RT was built with $\mathrm{BOMB}$ using the anilinylbenzyloxadiazole core and the structure of RT from the 1 s9e PDB file. ${ }^{22}$ The switch from the $1 \mathrm{rt} 4$ structure arose because the $1 \mathrm{~s} 9 \mathrm{e}$ structure has higher resolution (2.6 vs. $2.9 \AA$ ) and the $1 \mathrm{~s} 9 \mathrm{e}$ ligand is more similar to the U-Het-NH-PhX motif of the oxadiazole; it is a UHet1-L-Het2 member with Het1 as a pyridinone, $U$ as 3,5-dimethylphenoxy and L, a linking chain, as $\mathrm{CH}_{2} \mathrm{SCH}_{2}$. The structure of the $\mathbf{1}$ complex built using $B O M B$ is illustrated in Figure 3. It is similar to the structure in Figure 1 except for the conformation of the two methoxy groups. The conformation in Figure 1 generated by Glide with both methoxy groups in-plane and the one in Figure 3 from $B O M B$ with one almost perpendicular to the benzene ring differ in energy by only $0.16 \mathrm{kcal} / \mathrm{mol}$ for $o$-dimethoxybenzene at the MP2/6-311G** level. ${ }^{23}$

$B O M B$ was then used to build the 100 complexes corresponding to replacement of each phenyl hydrogen in the core with the 10 small groups. This revealed that the top- 25 scoring analogs are dominated by substitution of either chlorine or a methyl group at the 3-, 4-, and 2-position in the benzyl ring and at the 4-, 2-, and 3-position of the anilinyl ring in roughly that order. The top three analogs have chlorine at the 3-, 4-, and 5- position in the benzyl ring, where the methoxy groups in Figure 3 would be said to be at the 3- and 4-positions. Monte Carlo/freeenergy perturbation calculations in explicit water were also performed ${ }^{3}$ for mono-chlorination and favored substitution at the 2- and 6-positions of the benzyl ring and the 4-position of the anilinyl ring. The 45 possible dichloro-substituted compounds were built next with $B O M B$. The ten best scores are within $0.2 \log$ unit, but revealed a preference for chlorine at the 4position in the anilinyl ring and at the 5- and 6-positions in the benzyl ring.

Given this information, synthesis of compounds $\mathbf{1 1 - 2 0}$ in Table 1 was initiated. The table includes the $B O M B$ scores for $\mathbf{1}$ and the prepared compounds as well as the protein-ligand interaction energy for the complexes after conjugate-gradient optimization of the structures from $B O M B$ using $M C P R O^{24}$ with the OPLS-AA (protein) and OPLS/CM1A (ligand) force fields and a dielectric constant of 2 . At this point, it is noted that $\mathbf{1}$ scored poorly in comparison to the polychloro analogs; the difference was dominated by $\mathbf{1}$ being predicted to be less lipophilic by $1-2 \log$ units in $\mathrm{QP} \log \mathrm{P}_{\mathrm{o} / \mathrm{w}}(2.6 v$ s. 3.6-4.2) and for having 28 "bad" contacts vs. ca. 18 for the dichloro compounds. The additional undesirable contacts stem from the proximity of the methoxy oxygens and the aromatic and $\mathrm{C}_{\beta}$ carbons of Tyr181 and Tyr188. Furthermore, a global concern for the series was the proximity of the carboxylate oxygens of Glu138 to N4 of the oxadiazole and to the benzylic $\mathrm{CH}_{2}$ group.

\section{Synthesis and Assay Results}

The oxadiazole derivatives were prepared via cyclization of substituted phenylacetic hydrazides 25 and substituted phenyl isocyanide dichlorides, ${ }^{26}$ as illustrated in Scheme 2 for 14. The procedure finds precedent in the synthesis of $N, N$-diphenyloxadiazol-2,5-diamines from semicarbazides. ${ }^{27}$ Full synthetic and spectral details are provided in the Supporting Information. The assay results for the first three compounds that were prepared, 11-13, were hardly encouraging. $\mathbf{1 1}$ and $\mathbf{1 3}$ are benign - inactive and non-cytotoxic, and for $\mathbf{1 2}$ there was at best a small hint of activity above $10 \mu \mathrm{M}$ that was masked by the onset of cytotoxicity with a $\mathrm{CC}_{50}$ of $32 \mu \mathrm{M}$. It was also somewhat surprising that if $\mathbf{1 2}$ with a 3-chloro substituent in the benzyl ring were active, that the 3,5-dimethyl analog (13) showed no activity up to $100-\mu \mathrm{M}$. A 3,5- dimethylphenoxy group is present as the $\mathrm{U}$ group in the pyridinone ligand (a 2-nM NNRTI) in the $1 \mathrm{~s} 9 \mathrm{e}$ crystal structure, ${ }^{22}$ but it is clearly ineffective in the present oxadiazole series. 
Nevertheless, 14 was then prepared to probe the 2,6-dichloro pattern that was supported by the computational analyses and has precedent in the 2,6-difluoro and 2,6-dimethyl substitution for the $\mathrm{U}$ groups in $\mathbf{2}$ and $\mathbf{4} . \mathbf{1 5}$ was also prepared as a reference compound, though there was no hope for it as an active in view of the results for the dichloro-analogs $\mathbf{1 1}$ and $\mathbf{1 2}$. Fortunately, 14 was found to be a definite active at the $820-\mathrm{nM}$ level and a respectable low-molecular weight (355) lead for further optimization. Thus, $\mathbf{1}$ can be classified as a near-miss with need, at least, for an alternative substitution pattern in the benzyl ring. For reference, it is noted that the nucleoside inhibitor $\mathrm{d} 4 \mathrm{~T}$ and the NNRTI nevirapine are active at the 1.6- $\mu \mathrm{M}$ and $110-\mathrm{nM}$ levels in the MT-2 assay (Table 1).

\section{LT00141083 Interlude}

At this point, a paper appeared reporting results of another screening effort seeking NNRTIs. 28 In that case, a 3D-pharmacophore model was used for initial filtering of the Derwent and CAP databases, which was followed by docking of ca. 10,000 compounds with Glide. Six compounds were purchased to test their "in vitro RNA-dependent DNA polymerase activity of recombinant RT." Five of the six compounds were reported to be active. This includes a compound $\mathbf{5}$ in reference 28 that was drawn as being identical to the core in the present work, 16 in Table 1. No spectral or other characterization data were provided for the assayed compounds. ${ }^{28}$ Compound 5 in reference ${ }^{28}$ yielded an $\mathrm{IC}_{50}$ of $4.2 \mu \mathrm{M}$ in the enzyme assay; nevirapine was also reported as $0.18 \mu \mathrm{M}$ for reference, which is a little less potent than in our assay (Table 1). There was one available commercial source for 16, LaboTest AB, and the compound is designated LT00141083. We purchased it. The authors of the other study 28 verified that they had also purchased their compound 5 from LaboTest. A ${ }^{1} \mathrm{H}$ NMR spectrum was taken of the purchased sample, which immediately revealed that it could not be $\mathbf{1 6}$ in view of having the incorrect number of protons and, for example, no benzylic protons. Thus, $\mathbf{1 6}$ was then synthesized and submitted to the MT-2 anti-HIV assay. In view of the assay results for $11-15$ and the $B O M B$ scores, there was no possibility that $\mathbf{1 6}$ could be active. This was confirmed (Table 1). Under the circumstances, it seems highly unlikely that $\mathbf{1 6}$ was assayed in the other study. ${ }^{28}$ The ${ }^{1} \mathrm{H}$ NMR spectra of the purchased material and $\mathbf{1 6}$ are compared in the Supplementary Information.

\section{A Few More Compounds}

From the $B O M B$ analyses and visualization of the resultant structures, replacement of hydrogen by chlorine or a methyl at $\mathrm{C} 3$ in the anilinyl ring of $\mathbf{1 4}$ seemed worth investigation. However, it is noted that such additions break the symmetry of the para-substituted phenyl ring. This can have negative impact on binding if the added substituent in the bound state can only be at either effectively the 3- or 5- position, while it can sample both in the unbound state. This could lead to a factor of 2 reduction in binding affinity or perhaps even a factor of 10 in an extreme case in which the rotation of the phenyl ring is free in the unbound state, but restricted to a dihedral range of $36^{\circ}$ when bound. For the present case, substitution of chlorine or a methyl group at $\mathrm{C} 3$ or $\mathrm{C} 5$ appeared to be allowed sterically and yielded similar $B O M B$ scores, while a trifluoromethyl group is only allowed on the side facing the benzyl group in the bound state.

In the event, 17 and 19 were synthesized along with the trifluoromethyl analog 20. An additional product, 18, which arose in the course of failed attempts to synthesize the 3-methoxy analog, was also assayed. The methyl derivative $\mathbf{1 7}$ did not improve on the activity of $\mathbf{1 4}$, and 18 and 20 are inactive, but the chloro analog 19 is a 310-nM NNRTI (Table 1). There is clearly room for additional lead optimization in this series, but it has been demonstrated that a nearmiss from virtual screening can be evolved into a true active. Finally, in comparing the experimental activities, $B O M B$ scores and protein-ligand interaction energies $\left(\mathrm{E}_{\mathrm{XX}}\right)$ in Table 1 , correlations are not perfect. However, the compounds with $B O M B$ scores above -5.7 are all inactive. $\mathrm{E}_{\mathrm{XX}}$ is not a particularly good index of potential binding because it tends to increase 
with increasing size of the ligand; dividing it by the number of atoms or molecular weight is flawed without correction for parts of the ligand that make no contact with the protein.

Nevertheless, compounds with both very favorable $B O M B$ scores and $\mathrm{E}_{\mathrm{XX}}$ values are likely to be active. $\mathbf{2 0}$ is the exception, though it suffers from a symmetry loss of at least a factor of two in activity.

\section{Conclusions}

Virtual screening of the Maybridge library of ca. 70,000 compounds was performed using a similarity filter, docking, and MM-GB/SA post-processing to seek potential non-nucleoside inhibitors of HIV-1 reverse transcriptase (NNRTIs). The protocol did well in identifying known NNRTIs. Six representative, top-scoring compounds were purchased from Maybridge. Two were impure and gave incorrect mass spectra. After purification, the other four were submitted to an anti-HIV assay using infected human T-cells and found to show no ability to inhibit HIV replication. However, the highest-ranked library compound, the oxadiazole derivative $\mathbf{1}$, was subjected to further computational analysis to seek modifications that might yield an active anti-HIV agent. A small substituent scan was performed for the anilinylbenzyloxadiazole core with the $B O M B$ program, which specifically suggested removal of the methoxy groups in the benzyl ring and addition of two or three chlorines or methyl groups. Synthesis and assaying of several such compounds did yield active anti-HIV agents, as summarized in Table 1. The study demonstrates that it is possible to learn from a formally unsuccessful virtual-screening exercise and, with the aid of computational analyses, to evolve efficiently a false positive into a true active. The present case may be viewed as favorable in view of the availability of significant structure-activity data for NNRTIs; however, it provides a prototype for future efforts and, in fact, success was far from guaranteed owing to the sensitivity of activity to structure, the plethora of incorrect choices that could be made, and the lack of precedent for five-memberedring heterocycles as the central ring in NNRTIs. In addition, the obvious need for adequate structure validation of purchased compounds was confirmed by the apparent misrepresentation of a compound elsewhere as the present oxadiazole core, $\mathbf{1 6}$.

\section{Supplementary Material}

Refer to Web version on PubMed Central for supplementary material.

\section{Acknowledgments}

Gratitude is expressed to the National Institutes of Health (AI44616, GM32136, GM49551) for support. Computational assistance from Drs. Julian Tirado-Rives and Ivan Tubert-Brohman was also appreciated.

\section{References}

1. 2006 AIDS Epidemic Update. Geneva: UNAIDS; 2006.

2. (a) Kohlstaedt LA, Wang J, Friedman JM, Rice PA, Steitz TA. Crystal structure at 3.5 Å resolution of HIV-1 reverse transcriptase complexed with an inhibitor. Science 1992;256:1783-1790. [PubMed: 1377403] (b) Smerdon SJ, Jäger J, Wang J, Kohlstaedt LA, Chirino AJ, Friedman JM, Rice PA, Steitz TA. Structure of the binding site for nonnucleoside inhibitors of reverse transcriptase of human immunodeficiency virus type 1. Proc. Natl. Acad. Sci. U.S.A 1994;91:3911-3915. [PubMed: 7513427] (c) De Clercq E. New approaches toward anti-HIV chemotherapy. J. Med. Chem 2005;48:1297-1313. [PubMed: 15743172]

3. (a) Jorgensen WL, Ruiz-Caro J, Tirado-Rives J, Basavapathruni A, Anderson KS, Hamilton AD. Computer-aided design of non-nucleoside inhibitors of HIV-1 reverse transcriptase. Bioorg. Med. Chem. Lett 2006;16:663-667. [PubMed: 16263277] (b) Ruiz-Caro J, Basavapathruni A, Kim JT, Wang L, Bailey CM, Anderson KS, Hamilton AD, Jorgensen WL. Optimization of diarylamines as non-nucleoside inhibitors of HIV-1 reverse transcriptase. Bioorg. Med. Chem. Lett 2006;16:668-671. 
[PubMed: 16298131] (c) Thakur VV, Kim JT, Hamilton AD, Bailey CM, Domaoal RA, Wang L, Anderson KS, Jorgensen WL. Optimization of pyrimidinyl- and triazinyl-amines as non-nucleoside inhibitors of HIV-1 reverse transcriptase. Bioorg. Med. Chem. Lett 2006;16:5664-5667. [PubMed: 16931015] (d) Kim JT, Hamilton AD, Bailey CM, Domaoal RA, Wang L, Anderson KS, Jorgensen WL. FEP-guided selection of bicyclic heterocycles in lead optimization for non-nucleoside inhibitors of HIV-1 reverse transcriptase. J. Am. Chem. Soc 2006;128:15372-15373. [PubMed: 17131993]

4. (a) Blake JF, Laird ER. Recent advances in virtual ligand screening. Ann. Rep. Med. Chem 2003;38:305-314. (b) Leach AR, Shoichet BK, Peishoff CE. Prediction of protein-ligand interactions. Docking and scoring: successes and gaps. J. Med. Chem 2006;49:5851-5855. [PubMed: 17004700]

5. Barreiro G, Guimarães CRW, Tubert-Brohman I, Lyons TM, Tirado-Rives J, Jorgensen WL. Search for non-nucleoside inhibitors of HIV-1 reverse transcriptase using chemical similarity, molecular docking, and MM-GB/SA scoring. J. Chem. Info. Model. 2007in press.

6. Ren J, Esnouf RM, Hopkins AL, Warren J, Balzarini J, Stuart DI, Stammers DK. Crystal structures of HIV-1 reverse transcriptase in complex with carboxanilide derivatives. Biochemistry 1998;37:1439414403. [PubMed: 9772165]

7. Friesner RA, Banks JL, Murphy RB, Halgren TA, Klicic JJ, Mainz DT, Repasky MP, Knoll EH, Shelley M, Perry JK, Shaw DE, Francis P, Shenkin PS. Glide: A new approach for rapid, accurate docking and scoring. 1. Method and assessment of docking accuracy. J. Med. Chem 2004;47:1739-1749. [PubMed: 15027865]

8. (a) Jorgensen WL, Maxwell DS, Tirado-Rives J. Development and testing of the OPLS all-atom force field on conformational energetics and properties of organic liquids. J. Am. Chem. Soc 1996;118:11225-11236. (b) Jorgensen WL, Tirado-Rives J. Chemical theory and computation special feature: Potential energy functions for atomic-level simulations of water and organic and biomolecular systems. Proc. Nat. Acad. Sci USA 2005;102:6665-6670. [PubMed: 15870211]

9. (a) Still WC, Tempczyk A, Hawley RC, Hendrickson T. Semianalytical treatment of solvation for molecular mechanics and dynamics. J. Am. Chem. Soc 1990;112:6127-6129. (b) Jorgensen WL, Ulmschneider JP, Tirado-Rives J. Free energies of hydration from a generalized Born model and an all-atom force field. J. Phys. Chem. B 2004;108:16264-16270.

10. MacroModel, version 9.0. New York, NY: Schrödinger LLC; 2005.

11. (a) Lin TS, Luo MZ, Liu MC, Pai SB, Dutschman GE, Cheng YC. Antiviral activity of 2',3'-dideoxy$\beta$-L-5-fluorocytidine ( ${ }^{2}$-L-EddC) and 2',3'-dideoxy-2 ${ }^{2}$ L-cytidine ( ${ }^{2}$-L-ddC) against hepatitis B virus and human immunodeficiency virus type 1 in vitro. Biochem. Pharmacol 1994;47:171-174.

[PubMed: 8304960] (b) Ray AS, Yang Z, Chu CK, Anderson KS. Novel use of a guanosine prodrug approach to convert 2',3'-didehydro-2',3'-dideoxyguanosine into a viable antiviral agent. Antimicrob. Agents Chemother 2002;46:887-891. [PubMed: 11850281]

12. (a) Muraglia E, Kinzel OD, Laufer R, Miller MD, Moyer G, Munshi V, Orvieto F, Palumbi MC, Pescatore G, Rowley M, Williams PD, Summa V. Tetrazole thioacetanilides: Potent non-nucleoside inhibitors of WT HIV reverse transcriptase and its K103N mutant. Bioorg. Med. Chem. Lett 2006;16:2748-2752. [PubMed: 16503141] (b) Wang Z, Wu B, Kuhen KL, Bursulaya B, Nguyen TN, Nguyen DG, He Y. Synthesis and biological evaluations of sulfanyltriazoles as novel HIV-1 non-nucleoside reverse transcriptase inhibitors. Bioorg. Med. Chem. Lett 2006;16:4174-4177. [PubMed: 16781149] (c) De La Rosa M, Kim HW, Gunic E, Jenket C, Boyle U, Koh Y-H, Korboukh I, Allan M, Zhang W, Chen H, Xu W, Nilar S, Yao N, Hamatake R, Lang SA, Hong Z, Zhang Z, Girardet J-L. Tri-substituted triazoles as potent non-nucleoside inhibitors of the HIV-1 reverse transcriptase. Bioorg. Med. Chem. Lett 2006;16:4444-4449. [PubMed: 16806925]

13. Tirado-Rives J, Jorgensen WL. Contribution of conformer focusing to the uncertainty in predicting free energies for protein-ligand binding. J. Med. Chem 2006;49:5880-5884. [PubMed: 17004703]

14. Hann MM, Leach AR, Harper G. Molecular complexity and its impact on the probability of finding leads for drug discovery. J. Chem. Inf. Comput. Sci 2001;41:856-864. [PubMed: 11410068]

15. Rizzo RC, Udier-Blagovic M, Wang DP, Watkins EK, Kroeger Smith MB, Smith RH Jr, TiradoRives J, Jorgensen WL. Prediction of activity for non-nucleoside inhibitors with HIV-1 reverse transcriptase based on Monte Carlo simulations. J. Med. Chem 2002;45:2970-2987. [PubMed: 12086483] 
16. Blagović MU, Tirado-Rives J, Jorgensen WL. Validation of a model for the complex of HIV-1 reverse transcriptase with non-nucleoside inhibitor TMC125. J. Am. Chem. Soc 2003;125:6016-6017. [PubMed: 12785806]

17. Das K, Clark AD Jr, Lewi PJ, Heeres J, de Jonge MR, Koymans LMH, Vinkers HM, Daeyaert F, Ludovici DW, Kukla MJ, De Corte B, Kavash RW, Ho CY, Ye H, Lichtenstein MA, Andries K, Pauwels R, de Béthune M-P, Boyer PL, Clark P, Hughes SH, Janssen PAJ, Arnold E. Roles of conformational and positional adaptability in structure-based design of TMC125-R165335 (Etravirine) and related non-nucleoside reverse transcriptase inhibitors that are highly potent and effective against wild-type and drug-resistant HIV-1 variants. J. Med.Chem 2004;47:2550-2560. [PubMed: 15115397]

18. Pyrimidines such as $\mathbf{2} \mathbf{- 6}$ are in the class Het-NH-Ph-U in which the $\mathrm{U}$ group is now delivered into the aryl box formed by Tyr181, Tyr188, Trp229, and Phe227 from the "right" instead of from the "left" as in Figure 1 for $\mathbf{1}$.

19. Ludovici DW, De Corte BL, Kukla MJ, Ye H, Ho CY, Lichtenstein MA, Kavash RW, Andries K, de Béthune M-P, Azijn H, Pauwels R, Lewi PJ, Heeres J, Koymans LMH, de Jonge MR, Van Aken KJA, Daeyaert FFD, Das K, Arnold E, Janssen PAJ. Evolution of anti-HIV drug candidates. Part 3: diarylpyrimidine (DAPY) analogues. Bioorg. Med. Chem. Lett 2001;11:2235-2239. [PubMed: 11527705]

20. Heeres J, de Jonge MR, Koymans LMH, Daeyaert FFD, Vinkers M, Van Aken Koen JA, Arnold E, Das K, Kilonda A, Hoornaert GJ, Compernolle F, Cegla M, Azzam RA, Andries K, de Bethune MP, Azijn H, Pauwels R, Lewi PJ, Janssen PAJ. Design, synthesis, and SAR of a novel pyrazinone series with non-nucleoside HIV-1 reverse transcriptase inhibitory activity. J. Med. Chem 2005;48:1910-1918. [PubMed: 15771435]

21. QikProp, v 3.02006New YorkSchrödinger LLCQikProp is called as a subroutine by BOMB for each generated structure.

22. Himmel DM, Das K, Clark AD Jr, Hughes SH, Benjahad A, Oumouch S, Guillemont J, Coupa S, Poncelet A, Csoka I, Meyer C, Andries K, Nguyen CH, Grierson DS, Arnold E. Crystal structures for HIV-1 reverse transcriptase in complexes with three pyridinone derivatives: A new class of nonnucleoside inhibitors effective against a broad range of drug-resistant strains. J. Med. Chem 2005;48:7582-7591. [PubMed: 16302798]

23. Tsuzuki S, Houjou H, Nagawa Y, Hiratani K. The second stable conformation of the methoxy groups of $o$-dimethoxybenzene: stabilization of perpendicular conformation by $\mathrm{CH}-\mathrm{O}$ interaction. J. Chem. Soc. Perkin Trans 2002;2:1271-1273.

24. Jorgensen WL, Tirado-Rives J. Molecular modeling of organic and biomolecular systems using BOSS and MCPRO. J. Comput. Chem 2005;26:1689-1700. [PubMed: 16200637]

25. Alanine A, Anselm L, Steward L, Thomi S, Vifian W, Groaning MD. Synthesis and SAR evaluation of 1,2,4-triazoles as $\mathrm{A}_{2 \mathrm{~A}}$ receptor antagonists. Bioorg. Med. Chem. Lett 2004;14:817-821. [PubMed: 14741297]

26. Van Dort M, Neubig R, Counsell RE. Radioiodinated p-iodoclonidine. A high-affinity probe for the alpha.2-adrenergic receptor. J. Med. Chem 1987;30:1241-1244. [PubMed: 3037080]

27. Zinner G, Heitmann M. Arbimide and carbonyl cyclizations of semicarbazides. Arch. Pharm 1981;314:193-196.

28. Barreca ML, De Luca L, Iraci N, Rao A, Ferro S, Maga G, Chimirri A. Structure-based pharmacophore identification of new chemical scaffolds as non-nucleoside reverse transcriptase inhibitors. J. Chem. Inf. Model 2007;47:557-562. [PubMed: 17274611] 


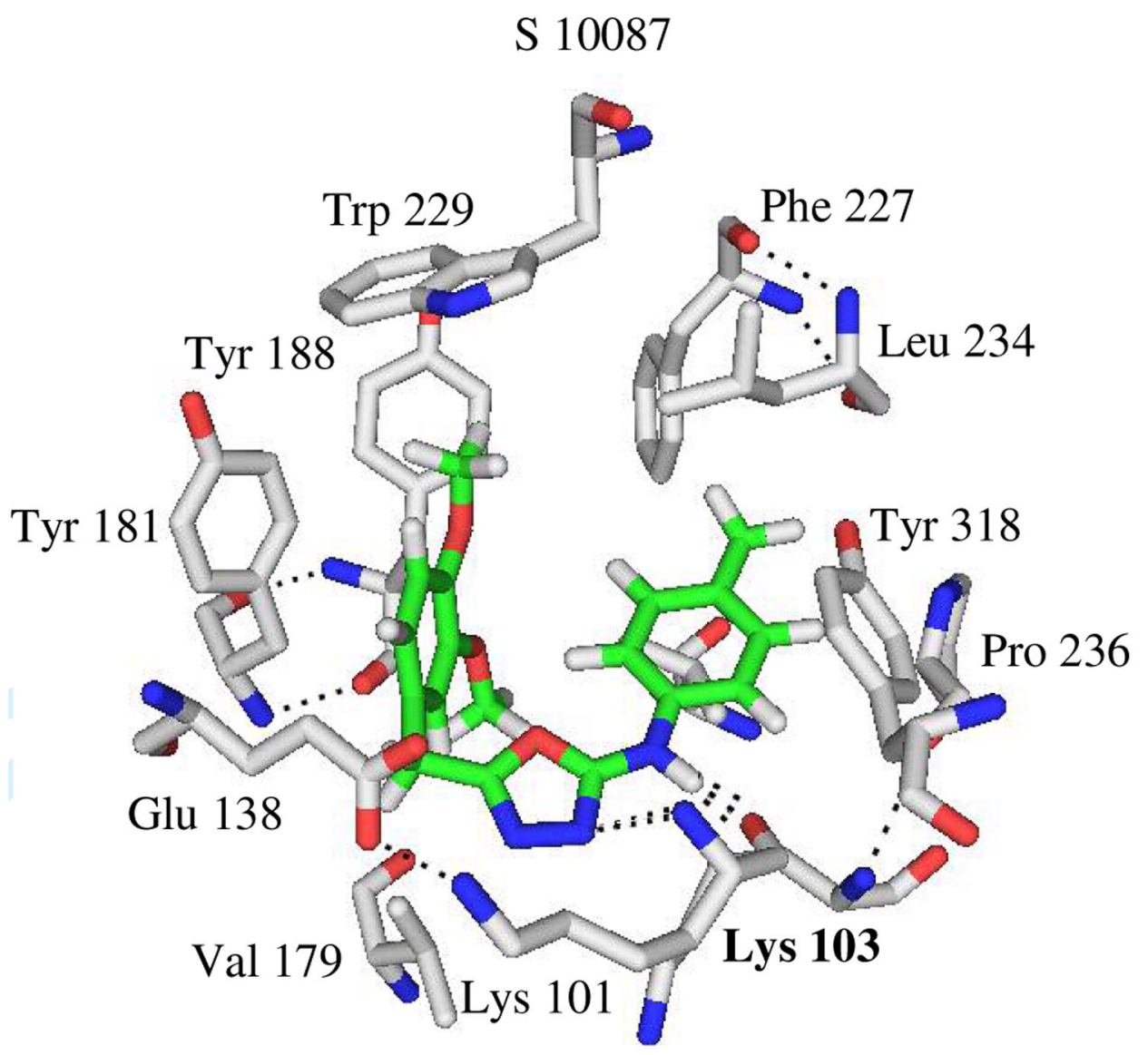

Figure 1.

Computed structure for $\mathbf{1}$ bound to HIV-1 reverse transcriptase from Glide docking followed by energy minimization with MacroModel. Only protein residues in the first layer around the ligand are illustrated. The ligand is positioned as expected from known structures such as for 4. 16,17 


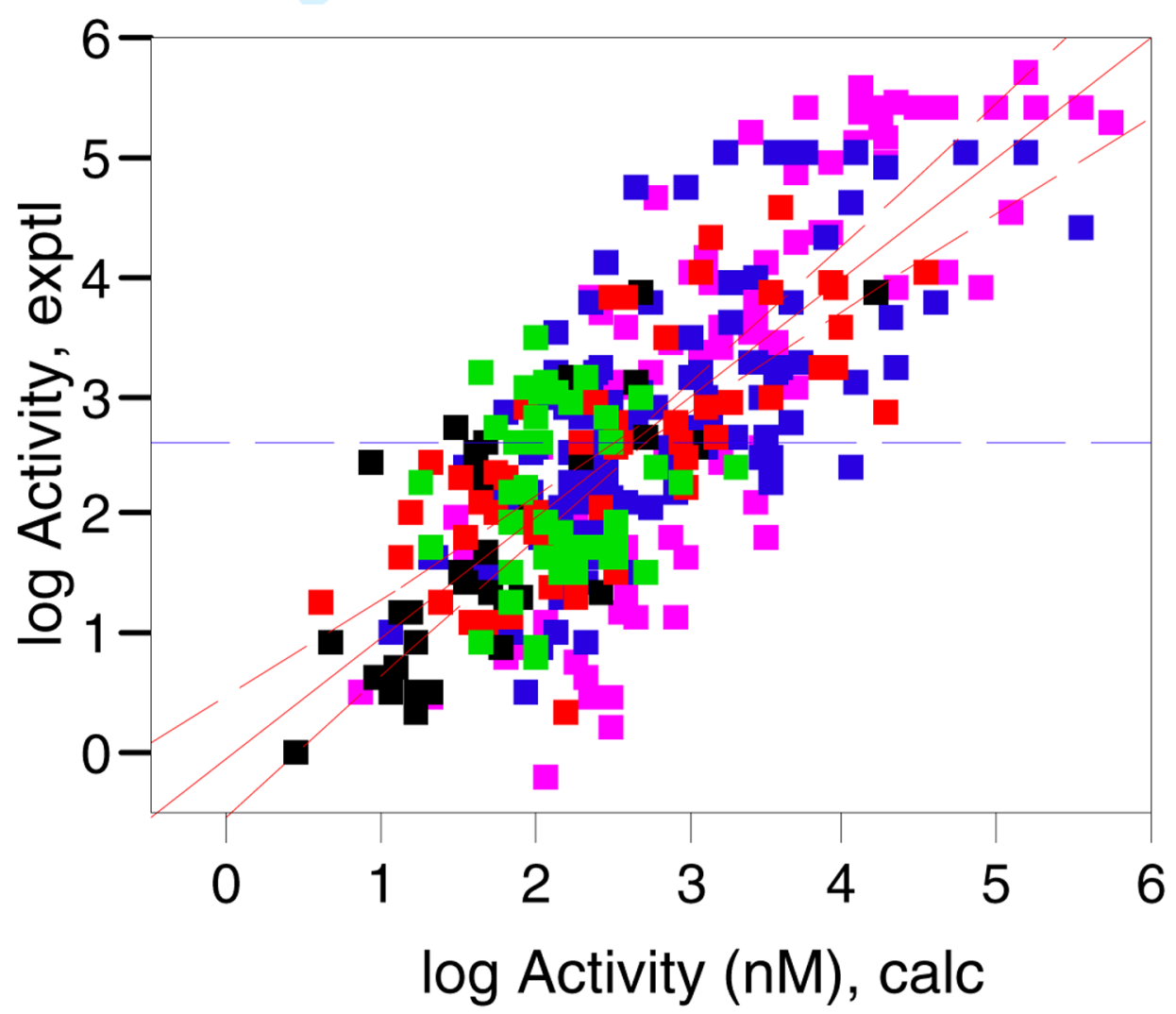

Figure 2.

Training results for scoring using $B O M B$ for 339 complexes with HIV-RT (magenta - uracil derivatives, green - analogs of 2), COX-2 (blue), FKBP (red), and p38 kinase (black). The correlation coefficient for the fit, $r^{2}$, is 0.58 and the rms error is $0.82 \log$ unit. Indicator variables are used to provide constant offsets for three datasets. 


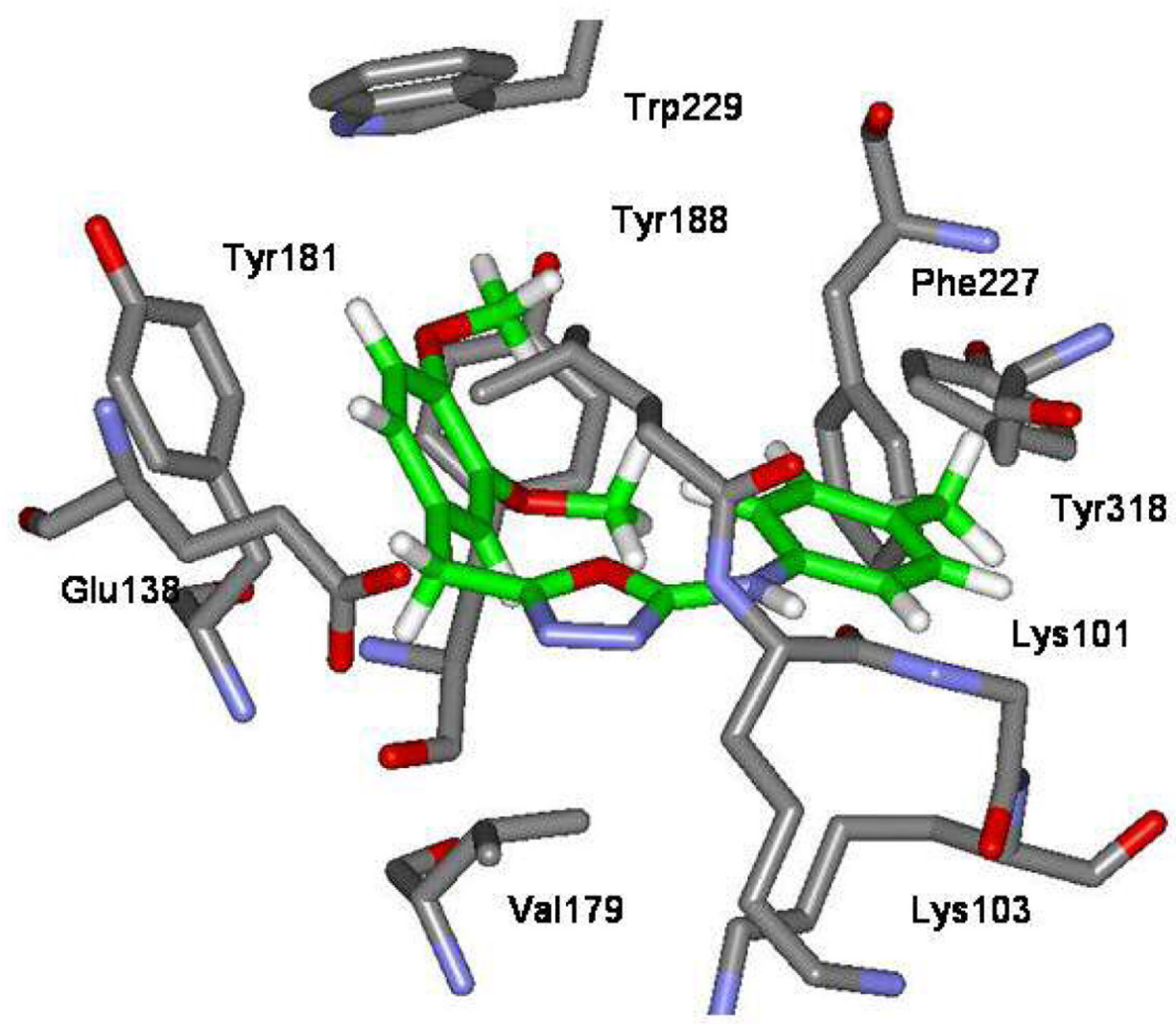

Figure 3.

Structure for the complex of $\mathbf{1}$ with HIV-RT as computed by BOMB. The 176 residues nearest the ligand are included in the calculations. 
<smiles>Cc1ccc(-n2c(C)nnc2SCC(=O)Nc2ccc(C)cc2Br)cc1</smiles><smiles>Cc1ccc(-n2c(C)nnc2SCC(=O)Nc2ccccc2)cc1</smiles><smiles>Cc1cc(C)c(-n2nnnc2SCC(=O)Nc2ccccc2Cl)c(C)c1</smiles>

Scheme 1.

Recent NNRTI Hits from HTS (Reference 12) 


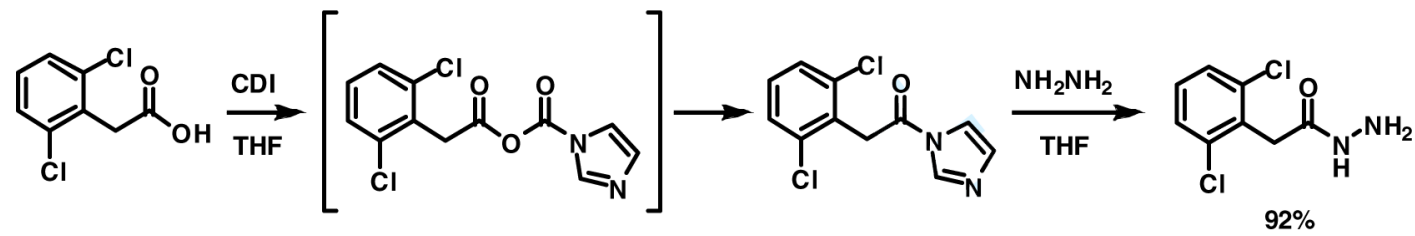

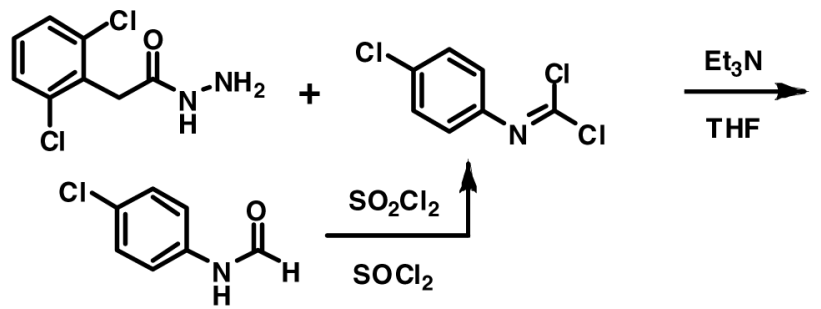<smiles>Clc1ccc(Nc2nnc(Cc3c(Cl)cccc3Cl)o2)cc1</smiles>

Scheme 2.

Synthesis of 2,5-oxadiazole derivatives. 


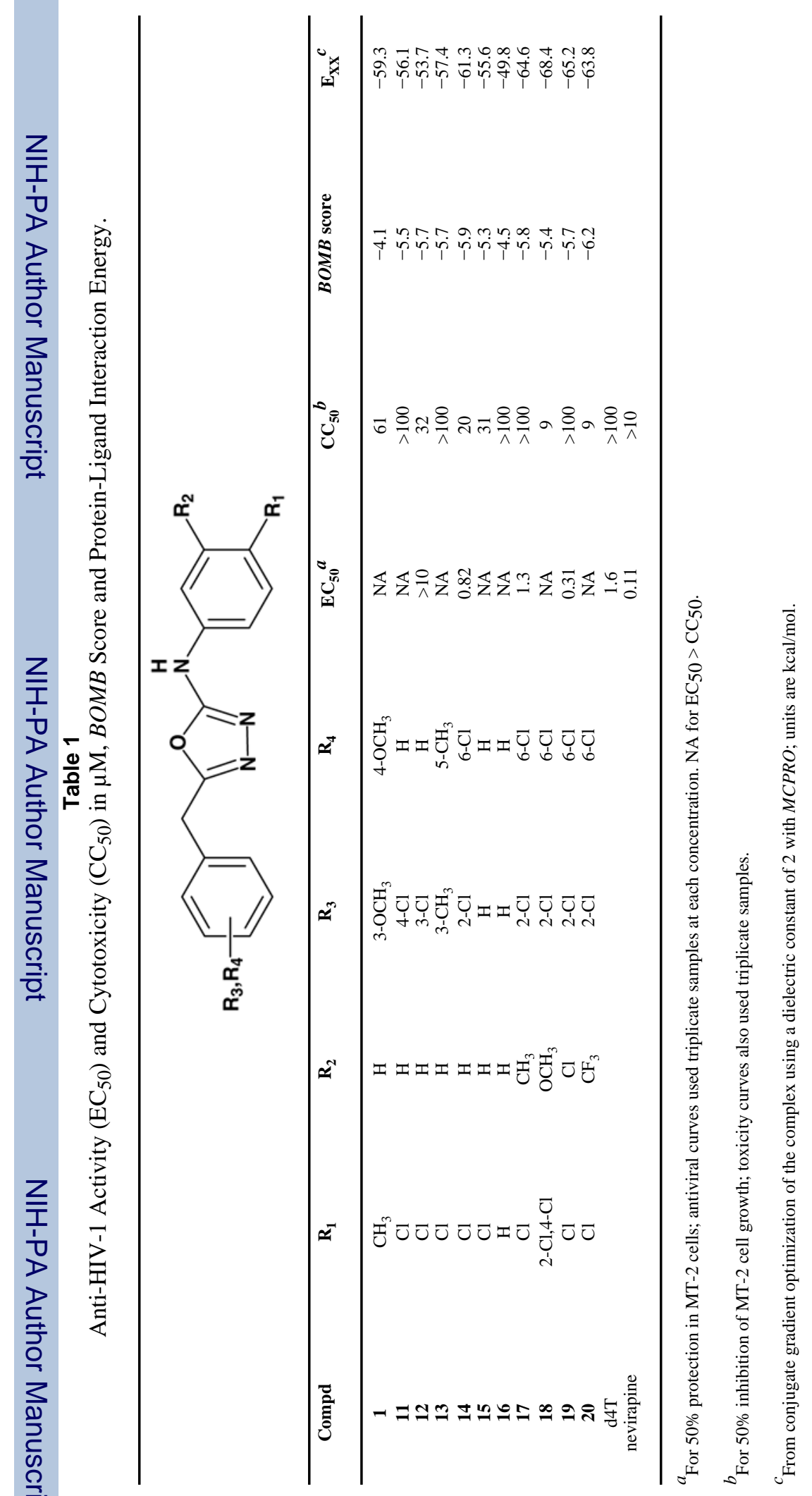

